\title{
Estimation of trade-offs with capture-recapture models: a case study on the lesser snow goose
}

\author{
ANNE VIALLEFONT ${ }^{1}$, EVAN G. COOCH ${ }^{2}$ \& FRED COOKE ${ }^{2},{ }^{1}$ CEFE/ \\ CNRS, Montpellier, France and ${ }^{2}$ Department of Biological Sciences, Simon Fraser \\ University, Canada
}

SUMMARY Trade-offs between traits such as fecundity or survival are fundamental to much of our understanding of the evolution of life histories. There has been much renewed interest and controversy concerning methods for estimating trade-offs, in the wild or in captivity, and with or without experimental manipulations. In this paper, we assess the general question of the utility of modern capture-recapture methods as a robust tool for estimating trade-offs in natural populations. We present results from analyses of two forms of trade-offs: the cost of present reproduction on future survival and the cost of present reproduction on the probability of breeding in the future. We apply the methods to data from a long-term study of a snow goose population, and generally discuss the advantages and potential problems with various approaches.

\section{Introduction}

Trade-offs among life history traits have been proposed as some of the most important factors in the evolution of life histories (e.g. Gadgil \& Bossert, 1970; Stearns, 1989; Jönsson \& Tuomi, 1994). Life history theory predicts a cost of reproduction on future reproductive events, or on future adult survival (Williams, 1966; Reznick, 1985). While the concept of trade-offs is virtually ubiquitous in most discussions of life history theory, detecting such trade-offs in the wild poses several difficulties: (1) environmental factors, or maternal inheritance, can induce a positive correlation between present and future reproductive success (or survival) (Van Noordwijk \& De Jong, 1986); (2) animals can achieve individual optima (Gustaffson \& Sutherland, 1988; Daan et al., 1990). Therefore, such trade-offs are rarely detectable in the wild without manipulations (Nur, 1990). While variation in fecundity is usually easy to assess (e.g. eggs are easy to count), assessment of 
trade-offs affecting future survival has often suffered from the use of inappropriate estimates of mortality rates (see Table 1). Capture-mark-recapture (CMR) models provide a powerful tool for rigorous estimation of local survival rates and formal treatment of trade-offs, in manipulated or non-manipulated populations. In some sense, trade-offs represent Markov processes such that events in year $t+x$ are conditional on events in year $t$. Recent extensions of CMR methodology, such as multi-state models, theoretically allow the examination of trade-offs in this context (Nichols et al., 1994). We review models permitting detection of such effects, and assess the cost of first reproduction in a population of lesser snow geese (Anser caerulescens) nesting at La Pérouse Bay, Manitoba, Canada, relative to the number of eggs laid at the first breeding occasion.

\section{Importance of trade-offs in the evolution of life histories}

In the wild, animals must allocate both their time and energy to several different activities (Principle of Allocation; Levins, 1968). Increase in fitness associated with selection acting on a given trait must have a cost in terms of another fitness component, otherwise both would increase indefinitely. The optimal decision is then to allocate one's time and energy so that the maximum possible fitness is achieved.

The origin of such trade-offs can be physiological (Sibly \& Calow, 1986) or genetic (animals having different selected strategies). There are at least 45 defined trade-offs (Stearns, 1992, p. 72), among which the most frequently studied are: cost of reproduction on adult survival; cost of reproduction on future reproductions; cost of reproduction on growth or condition; cost of number of offspring on quality of offspring (Stearns, 1992, p. 72). We are concerned in this study with the evidence for costs of reproduction on future reproduction or survival in animal populations, measured through phenotypic correlations between life history traits.

\section{Difficulties in measuring trade-offs}

Trade-offs are quite difficult to measure in the wild, due to environmental interactions that can lead to the observation of a positive correlation between life history traits (Van Noordwijk \& De Jong, 1986). Natural populations are often constituted of animals of different 'qualities' (due to environmental background, maternal effect), the 'good' animals doing better than the 'bad' ones in several traits. For this reason, Nur (1990) suggested that manipulative studies are a better way to assess such costs in bird populations: by manipulating the clutch size, or the brood size (for examples) of the birds, their reproductive effort is modified independently of their quality, therefore breaking the correlation between quality and the studied phenomenon. One objection to the manipulative approach to trade-offs is that such a method only reveals 'potential' trade-offs (the reason why animals don't take the risk of raising as many young as they can every year), whereas non-manipulative studies deal with 'actual' trade-offs (what really happens when they take the risk, and what the different strategies are in the population). Actual trade-offs are certainly rarer than potential ones, because animals regulate their own reproductive success to avoid such costs, and because only one strategy is likely to be favoured by natural selection. It is also more difficult to detect, because we then deal with much more subtle effects.

An additional problem is that, in small organisms with high metabolic rates 
('income breeders'), energy turnover may be too rapid for a trade-off to be detected in the long time interval that exists between samples, e.g. between reproduction and survival or reproduction in the following months/years (Sibly \& Calow, 1986).

The other practical difficulty in measuring trade-offs is the availability of methods for measuring survival in the wild: except in captivity, we can never be sure that we capture every animal that is alive one year after the observation or manipulation of their reproductive success. The most usual method consists in measuring survival as the proportion of animals caught or seen one year after the reproductive event under interest (return rate). This method has the advantage of applying to relatively short-time experiments (2 years of field work are sufficient to yield results), but is has important drawbacks often neglected in the literature. First, return rates are not survival rates, as they include the probability of coming back to the breeding site, and the probability of being caught or seen. The only exception to this is when the probability of capture of a living animal is $100 \%$ (as, for example, in Bradley et al., 1989), but this assumption must be checked carefully. Thus, return rates should not be used in place of true survival; for example, in Leslie matrix models or comparisons between species, sexes or any kind of groups likely to have different capture rates. Return rates only allow us to approximately assess differences in survival of animals from different treatments or groups having similar catchability (e.g. Török \& Toth, 1990; Orell \& Koivula, 1990). Moreover, when only return rates are measured, we cannot unambiguously distinguish between a cost of reproduction on next survival, and a cost on the probability of being on the breeding site the next year. This may be especially true in long-lived species (the 'capital breeders' of Sibly and Calow (1986)), where the cost of reproduction may be expressed as a temporary avoidance of breeding rather than an increased probability of death.

This problem has been largely underestimated by field biologists in the past. For example, among 28 manipulative or non-manipulative field studies of phenotypic correlations between fecundity and parental survival in vertebrates, listed in Appendix 2 of Stearns' book (1992), only one used a CMR model to estimate survival rates. Among the 26 studies that used return rates, only four checked that the capture rate was $100 \%$, or corrected for animals captured after several years of study. The 22 remaining studies, among which nine concluded existence of a cost of reproduction on survival, did not adequately address this question.

Several recent advances in CMR methodology enable us to determine simultaneously which factors significantly affect survival or capture rates. The most significant effects are detected by careful modelling and hypothesis testing (Lebreton et al., 1992). Until recently, many of these approaches have been avoided by evolutionary ecologists, probably because they were unaware of the power and flexibility of modern CMR techniques (but see Blondel et al. (1992) and Weimerskirch and Jouventin (1987)). We show below which capture-recapture models should be used to assess costs of reproduction on survival or on future reproduction, depending on the design of the study (see also Table 1).

\section{Estimating trade-offs between reproduction and survival}

Variation in survival among animals can be precisely modelled, and attributed to biological factors like age (Pollock, 1975, 1981), sex (e.g. Lebreton et al., 1992), control/treatment group (e.g. Burnham et al., 1987; Blondel et al., 1992; Skalski 
TABlE 1. Types of trade-offs measurable with CMR methods, and their corresponding modelling

\begin{tabular}{|c|c|c|}
\hline Trade-off & Corresponding model & References \\
\hline $\begin{array}{l}\text { Reproduction (manipulation or recorded } \\
\text { performance)/survival }\end{array}$ & $\begin{array}{l}\text { Group effect on survival } \\
\text { rate }\end{array}$ & Blondel et al. (1992) \\
\hline Reproduction (idem)/philopatry & $\begin{array}{l}\text { Group effect on survival } \\
\text { rate }\end{array}$ & Danchin \& Monnat (1992) \\
\hline Reproduction (idem)/senescence & $\begin{array}{l}\text { Group/age effect on } \\
\text { survival rate }\end{array}$ & Loison (1995, p. 194 sqq.) \\
\hline $\begin{array}{l}\text { Reproduction/next reproduction } \\
\text { (breeding probability) }\end{array}$ & 'Trap-shyness' & Gonzalez-Davilà (1994) \\
\hline First reproduction/survival & $\begin{array}{l}\text { 'Age' effect (two classes) } \\
\text { on survival rate }\end{array}$ & Viallefont et al. (1995) \\
\hline $\begin{array}{l}\text { First reproduction/next reproduction } \\
\text { (breeding probability) }\end{array}$ & $\begin{array}{l}\text { 'Age' effect (two classes) } \\
\text { on capture rate }\end{array}$ & Viallefont et al. (1995) \\
\hline Reproduction/next reproduction (quality) & Multi-stage models & Nichols et al. (1994) \\
\hline $\begin{array}{l}\text { Number of young produced/survival } \\
\text { of the young }\end{array}$ & $\begin{array}{l}\text { Recruitment models, } \\
\text { group effect }\end{array}$ & None known to us ${ }^{a}$ \\
\hline
\end{tabular}

${ }^{a}$ For recruitment models, see Clobert et al. (1994) and Pradel (1996).

et al., 1993) or environmental variables (Lebreton et al., 1992). In the study of trade-offs, relevant factors may include sex, when females incur a greater cost of reproduction than males, because their effort is more important (Trivers, 1972), or age, when particularly young (Newton, 1988) or old animals can incur a greater cost of breeding. Viallefont et al. (1995) assessed the cost of first-time breeding in a population of lesser snow geese, and did not detect lower survival after the first recorded breeding than after later breeding.

In manipulative studies, or when the natural success of animals is recorded in the wild, we can assess the relationship between membership in a group, usually noted $g$, and survival $\left(\phi_{g}\right)$. In this case, we can distinguish between an effect on immediate survival (first year after manipulation) or on later survival. We can also test for a trend among groups, when more than two levels of manipulation (or natural success) are recorded, as in Török and Toth (1990; 5 levels of manipulation, ranging from “ -4 or -5 ” to “ +4 or +5 ” nestlings). The importance of those effects can be tested across years, and when annual variations in survival are significant (for environmental reasons), additive models (Pradel et al., 1990) allow tests of the importance of interactions (e.g. whether a certain category of animals is more fragile than another in 'bad' years).

Multi-state CMR models (Arnason, 1973; Nichols et al., 1994) enable us to classify the animals into discrete states that can change across capture occasions. For example, in our case a bird can be classified as 'good' one year (when it lays a number of eggs well above the average that year) and 'medium' or 'poor' the next year. Nichols et al. (1994) have proposed such a study on meadow voles (Microtus pennsylvanicus), captured as breeders or non-breeders. Those models allow the estimation of survival probability of individuals according to their state the year before. This method permits the combination of data for several years to detect variation in survival for individuals that belong to a particular state, even when the animals change states between capture events. It should become a standard 
method for the measure of trade-offs between reproduction and survival in non-manipulated populations.

\section{Estimating trade-offs between present and future reproduction}

The cost of reproduction may result in a reduced reproduction the following year, or more generally at the next breeding occasion. This effect can be (1) qualitative, in the sense that the probability of breeding itself is affected, or (2) quantitative, in the sense that the level of reproduction is affected.

In estimations of survival using CMR models, capture probabilities are usually considered a nuisance factor, and modelled only to reduce bias and increase precision on the more important survival estimates. However, variations in the capture probability can have a significant biological meaning. Lower capture probability for a category of birds may reflect a tendency to quit the area temporarily, or to spend a shorter time in the capture area. When breeding birds are captured, reduced catchability can reflect a tendency to skip one breeding event, or a higher probability of failure in a subgroup of the population. For example, birds that nest only in alternate years have a smaller probability of capture after a successful breeding event, which appears as trap-shyness in the modelling of capture rates (Gonzalez-Davilà, 1994, Chapter 4). In a population of greater flamingos (Phoenicopterus ruber), Cézilly et al. (1996) explain the lower catchability of females (as compared to males) by their lower probability of renesting after a failed nesting attempt.

Viallefont et al. (1995) showed that female lesser snow geese tend to skip one reproductive event after their first successful breeding. In analyses of CMR data, this produces an apparent effect of 'age' on the recapture rate of those birds ('age' meaning here 'time elapsed since first breeding'). In this paper, we assess the magnitude of this effect as a function of the clutch size at first breeding (i.e. does the probability of skipping a breeding event depend on the clutch size laid at the first breeding attempt, as well as the age of first breeding?).

In manipulative studies, we can look for an effect of group on the capture rate the next year, and then we can differentiate between a cost of reproduction on survival or on the probability of breeding the next year.

The ideal tool to assess quantitative costs of reproduction in the wild is the multi-stage capture-recapture method (Nichols et al., 1994; Kendall \& Nichols, 1995). This method permits detection of differences in the probability of becoming a 'good' or a 'bad' reproducer, conditional on the performance of the animal at the previous capture occasion. Several years of data can be pooled to estimate the parameters, to enhance power or to assess the effects of temporary environmental changes. Using multi-stage models may allow detection of very subtle costs of reproduction, which would not be detectable with single-stage models, let alone with simple return rates.

\section{An example: assessing the costs of reproduction in the lesser snow goose}

6.1 Cost of first breeding on next reproduction, according to the performance at first breeding

Here we present a non-manipulative study that exemplifies the wide flexibility of the single-state models, and the diversity of the questions that can be assessed 
through these methods. Viallefont et al. (1995) studied the cost of first breeding in the lesser snow goose colony at La Pérouse Bay, and showed that birds breeding for the first time have a $23 \%$ lower probability of breeding the next year than more experienced birds. They also showed that this phenomenon is more important for birds starting to breed at a younger age: birds starting to breed at age 2 had a $45 \%$ probability of not breeding the following year, even though they were alive, while for birds starting their reproductive life at age 3 or older, this probability was $16 \%$. In the current study, we extend our analysis to determine if the probability of breeding one year after the first successful breeding depends on the clutch size laid in the first year. Two contrasting predictions can be made: (1) birds laying large clutches can incur a greater cost of reproduction and be less able to breed the next year, or (2) birds laying large clutches are in good condition and will have a greater probability of breeding the next year. The direction of the trend among groups should help us to distinguish between these two possibilities.

6.1.1 The data. The data consist of sighting histories of 1369 'young' female birds over 18 years. These birds were seen on nest for the first time at or before 4 years of age, or their resumed minimum age was 2 or 3 years. For all those birds, the clutch size at first sighting $c l$ was recorded, and classified as 'small' (409 birds), 'medium' (665 birds) or 'large' (295 birds), depending on the magnitude of the standardized deviation (SD) of the clutch size from the average clutch size for that year and age, $m_{y}$ : 'large clutches' are defined by $c l>m_{y}+2 / 3 \mathrm{SD}$ and 'small clutches' by $c l>m_{y}-2 / 3$ SD. Rockwell (1989) showed that in this species, clutch size is a good estimate of female reproductive effort. Snow geese rear self-feeding young, and most of the vigilance during the brood-rearing period is undertaken by the male of the pair. Thus, most of the costs of breeding experienced by the female are reflected in variation in the number of eggs laid.

Since we are using data from resightings on nest as our primary sample, there is a strong heterogeneity in the data: some birds are less visible than others because they regularly occupy a more remote site in the colony. The birds tend to occupy the same nest sites (or in the same area) every year, and the field workers also tend to search the same areas every year (with some adaptation early in the season). Thus, some birds have a capture history consisting almost only of 'ones' (records of presence), while others have a very low catchability. The most appropriate way to handle this in the analyses is to include in the models a 'trap-happiness' effect on recapture probabilities. This effect assumes that a bird that was caught in year $i$ is more likely to be caught in year $i+1$ than a bird that was missed in year $i$. Modelling of this effect in SURGE was proposed by Pradel (1993) and it is usually denoted $m$.

6.1.2 Modelling. The first model, tested independently for each group, is $\phi_{t \star F}, p_{t \star F \star m}$, where $\phi$ represents survival, $p$ capture probability, $t$ annual variation, $F$ the effect of first-breeding and $m$ the effect of potential trap-happiness (i.e. a bird that has been captured the year before has a higher probability of capture than a bird that has been missed the year before; this effect, also denoted 'immediate trap effect on capture' (ITEC), is described by Pradel (1993)). The notations * and + , proposed by Lebreton et al. (1992) from the classical notations in generalized linear models and ANOVA, stand for models with or without interactions, respectively. The models without interaction are also called 'additive models'.

We tested the GOF of the first model following the procedure described by 
Viallefont et al. (1995, Appendix A), modified for trap effect. The results of these tests appear in Table 2. We note that in this very general model, recapture one year after first breeding is estimated independently from that after a later breeding event, taken as reference. Since the effects of first-breeding and trap-happiness are contradictory, they are likely to compensate each other, and they are not independently estimable in models with interaction. Only additive models will allow us to evaluate the importance of the first-breeding effect, assuming that trap-happiness is the same for all birds.

TABle 2. GOF test for model $\phi_{t \star F}, p_{t \star F \star m}$, for young birds laying small, medium or large clutches in their first reproduction. The GOF test for such a model consists of three components (Viallefont et al., 1995, Appendix A): A = RELEASE GOF test of CJS model on data for the first capture (and first recapture) of the birds; $\mathrm{B}=$ RELEASE GOF test of CJS model on data for later recaptures-here the component 2.Ct of the RELEASE tests is not taken into account, to allow for trap dependence (Pradel, 1993); C = test of the equality of parameters after second capture, among the two above subsets

\begin{tabular}{lrrr}
\hline & $\chi^{2}$ OBS & df & $P$-level \\
\hline Small clutches & & & \\
A & 32.95 & 25 & 0.132 \\
B & 46.05 & 45 & 0.429 \\
C & 21.15 & 30 & 0.883 \\
Total & 100.15 & 100 & 0.477 \\
Medium clutches & & & \\
A & 26.32 & 29 & 0.608 \\
B & 64.87 & 46 & $0.035^{a}$ \\
C & 18.41 & 30 & 0.951 \\
Total & 109.61 & 105 & 0.360 \\
Large clutches & & & \\
A & 30.17 & 25 & 0.218 \\
B & 39.88 & 38 & 0.386 \\
C & 21.34 & 30 & 0.877 \\
Total & 91.39 & 93 & 0.528 \\
\hline
\end{tabular}

\footnotetext{
${ }^{a}$ Two out of 12 components of test $2 . \mathrm{Cm}$ with few data were significant, due to heterogeneity in the data. There was no systematic trend among the tests, and none of those components was found significant by Fisher's exact test.
}

From the starting model, the modelling was done independently for each clutch size grouping, and successive hypotheses of equality between parameters were tested by likelihood ratio tests (LRTs) between models, as described by Lebreton et al. (1992). For each group, the same model was selected as the most parsimonious (Table 3). This model, denoted $\phi, p_{t+F+m}$, contained a constant survival rate, and additive effects of time, trap-happiness and first-breeding effect on capture rates. We used a log link function in the additive constraint, in order to be able to express the effects found in terms of proportions (Viallefont, 1992).

Constant $\phi$ means that there is no significant effect of first-time breeding or of annual variation on survival, for any clutch size class. On average the survival was 


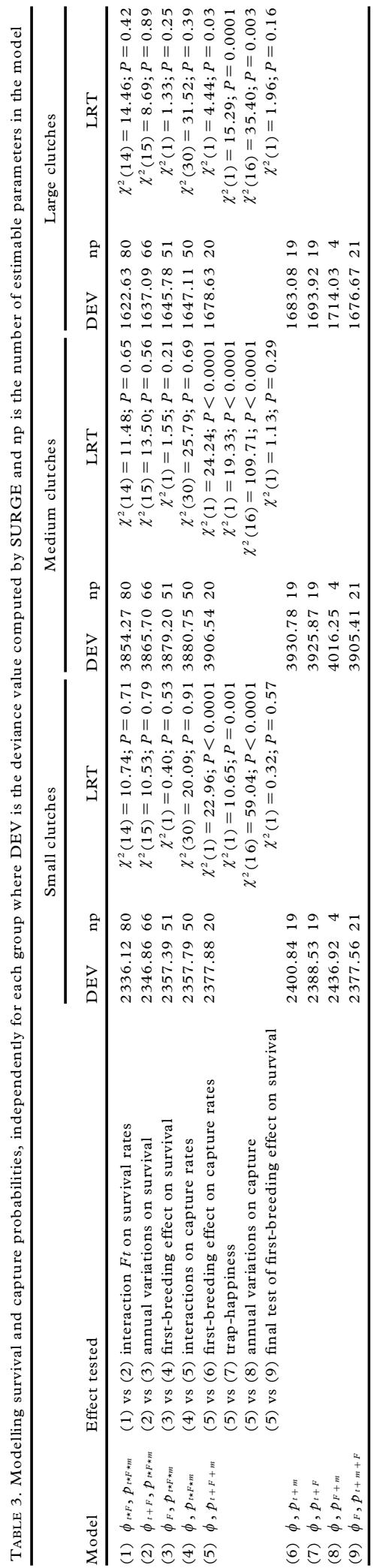


about $78 \%$ in each group, which agrees with previous results (e.g. Viallefont et al., 1995).

There was significant annual variation in capture rate, due mainly to variation in capture effort. There was also a significant trap-happiness effect, denoted $m$, due mainly to the fact that birds were faithful to a particular nesting area, and were thus likely to be resighted several years in a row. The term $F$ stands for the effect of first-time breeding: whatever their clutch size at their first recorded breeding, the birds are less likely to be seen the year immediately after. This corresponds to the findings by Viallefont et al. (1995) on recapture data. In this study the estimated probability of breeding the year immediately after the year after first breeding was $21.55 \%, 26.23 \%$ and $17.98 \%$ lower than for later occasions, for birds laying small, medium or large clutches, respectively.

This effect is compensated for by trap-happiness in the three data sets: the capture probability after a capture is increased by $31.78 \%, 30.58 \%$ and $45.43 \%$ for birds laying small, medium or large clutches, respectively, compared to the capture probability after a non-capture. This additive combination of the two effects (trap-happiness and first-time effect) means that a bird that has been seen in year $i$ is more likely to be seen again in year $i+1$ (apparent 'trap-happiness', due to its faithfulness to its nesting site for example), except if year $i$ was its first breeding occasion (first-breeding effect, leading to a smaller catchability in year $i+1$ ).

6.1.3 Comparison between clutch-size groups. We then modelled the data of the three clutch-size groups together, in order to assess between-group differences. We started this modelling from model $\phi_{c l \star F}, p(t+F+m) \star c l$, where $c l$ represents the clutch size category at the first recorded breeding. We then progressively accepted simplifying hypotheses, producing even simpler models (Table 4). The most accurate model supported by the data was $\phi, p_{t+F+m}$. Clutch size had no significant effect on survival, either in the first year or later. The mean survival in this population is estimated at $0.796\{0.772 ; 0.799\}$.

There was also no significant effect of the first clutch size, or of the interaction 'clutch-size-first-breeding' (noted $\mathrm{Fcl}$ ), on capture rate. This interaction is the actual effect we want to test, since it is the effect of clutch size on the first recapture probability, and we do not expect an effect of clutch size on later recaptures. The lack of significance of clutch size or interaction between clutch size and first-breeding effect suggests that the cost of reproduction after the first year is independent of how many eggs were laid at first breeding. The estimated probability of skipping one reproductive year after the first recorded attempt was $22.7 \%\{17.2 \% ; 27.8 \%\}$.

6.1.4 Power of the test. Failure to detect an effect of clutch size might be due to either a real lack of an effect or an inadequate sample size. Therefore, we assessed the power of the test for differences among groups in the $F$ effect, that is, the probability of detecting a difference between groups, given that a real difference exists. In our analysis, the test corresponding to this effect is the LRT between models $\phi, p_{t+F+m+F c l}$ and $\phi, p_{t+F+m}$. For practical reasons, we chose to ignore the trap-happiness in this computation, and assumed a sighting rate intermediate between those observed for animals having been seen or not the year before. We used the PROC SIMULATE EXPECT option of program RELEASE to generate 


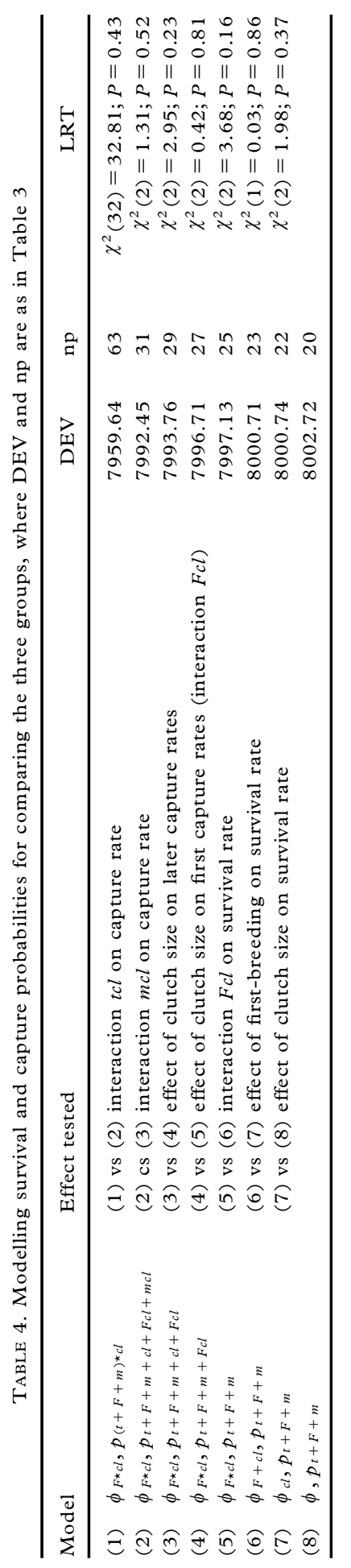


the expected data under the alternate hypotheses $\mathrm{H} 1$ and $\mathrm{H} 2$, where:

H0: model $\phi, p_{t+F}$, where $F$ represents a $23 \%$ drop in catchability 1 year after first breeding, no matter what the first clutch size was.

H 1: model $\phi, p_{t+F+F c l}$, where the drop in catchability after first breeding is $26 \%$ if the first clutch was large, $23 \%$ if it was medium and $20 \%$ if it was small.

H2: model $\phi, p_{t+F}+F c l$, where the effect of clutch size is much more important, the drop in catchability being $33 \%$ after a large first clutch, $23 \%$ after a medium one and $13 \%$ after a small one.

We then used SURGE to calculate the expected deviances under such models, and thus the coefficient of non-centrality, $\lambda$, of the $\chi^{2}$ LRT (Lebreton et al., 1992). From this, we obtained the power $\mathrm{P}$ of the test, given its first type error $\alpha$, its number of degrees of freedom (2) and its non-centrality coefficient $\lambda$.

The detectability of a small effect of clutch size on $F$ effect is very low ( $\mathrm{H} 1$ vs $\mathrm{H} 0, \lambda=0.47, \mathrm{P}=0.087$ for $\alpha=0.05, \mathrm{P}=0.155$ for $\alpha=0.1$ ). To improve this, more individuals, or a better capture rate, would be necessary. For example, if there were 10 times more individuals, $\lambda$ would be multiplied by 10 , and we would get $\mathrm{P}=0.482$ for $\alpha=0.05, \mathrm{P}=0.608$ for $\alpha=0.1$.

A more important effect of first clutch laid is, of course, much easier to detect: $\mathrm{H} 2$ vs $\mathrm{H} 0, \lambda=5.26, \mathrm{P}=0.526$ for $\alpha=0.05, \mathrm{P}=0.649$ for $\alpha=0.1$. If there were twice as much data, we would get $\mathrm{P}=0.835$ for $\alpha=0.05, \mathrm{P}=0.901$ for $\alpha=0.1$, which is a very good power. In this study, we can conclude that the effect of clutch size, if any, is smaller than the one assumed in hypothesis H2.

\subsection{Assessing the cost of reproduction after each occasion}

Findlay and Cooke (1987) found significant repeatability of clutch size in this population, which suggests that we should expect a positive correlation between clutch size across years, when using multi-stage CMR models. On the other hand, there could be a cost of laying numerous eggs that would counterbalance this effect and the birds would then alternate good and poor reproductive seasons. We tried to assess such a phenomenon with the use of multi-stage CMR models on adult birds (their sighting histories starting at age 4 or older.)

The modelling with multi-stage models starts by testing the GOF of a starting model, usually the historical model of Arnason (1973) and Schwarz et al. (1993), a straightforward generalization to several sites of the Cormack-Jolly-Seber model. The computer program MSSURVIV (Hines, 1994) allows the GOF testing of this model. The main problem is that the number of parameters in this model increases very rapidly with the number of occasions and especially with the number of categories. In our case (18 occasions, three 'states'), the model contained 201 parameters. Since the sighting rate is very low (only 830 birds out of 2803 have their clutch size recorded 2 or more years in a row), many of those parameters are not estimable. In addition, the data contain significant 'trap-dependence' (see earlier), precluding GOF of the Arnason and Schwarz model. In fact, the GOF test of such a model is $\chi^{2}(1485)=1594.5(P=0.0237)$. We could, of course, attempt to improve the fit of the general model by including trap-happiness in the parameterization. However, this would likely produce too many parameters to estimate them with any precision.

This absence of results points out that addressing more and more precise 
questions about the reproductive life of the animals necessitates more and more data. In our case, as probably in most population studies, we have more data about the presence/absence of an individual as a reproducer than about its particular reproductive success in a given year. Thus estimating survival rates, and even a cost of reproduction detectable as an absence from the breeding population, is feasible, whereas assessing individual transitions from one 'quality' group to another is impossible. The low sighting rate is just modelled and estimated in the first case, whereas in the second case it becomes a major handicap, as a good longitudinal follow-up of the individuals would be necessary. It seems that we have here reached the limits of the data set, rather than the limits of those models. However, we believe that such problems exist in numerous data sets, and that getting sufficient data for assessing such subtle effects is very demanding.

\section{Discussion}

The recent improvements towards flexibility and precision in CMR methodology now permit assessment of the costs of reproduction on both survival and next reproduction, on manipulated or non-manipulated wild populations. The methods proposed here can be used without any a priori hypotheses about the results, and all the effects listed in Table 1 can be combined to model more faithfully the complexity of natural systems than has been previously possible. For an example of such study with numerous effects combined, see Clobert et al. (1988). The possibility of testing additive effects of factors on the parameters (i.e. getting rid of the numerous interaction terms as in ANOVA) is especially useful when manipulations are impossible, there are not enough data or the capture rate is low. Even when there is annual variation in survival or capture rates, additive models allow the detection of rather small effects by a LRT with only one or two degrees of freedom, therefore with more power. Viallefont et al. (1995) provide an example of the use of such additive models, by comparing models $\phi, p_{t+F}$ and $\phi, p_{t}$ by a LRT with only one degree of freedom (thus with good power), to show evidence for a cost of first-time breeding in female lesser snow geese. This phenomenon would have been impossible to detect in the short term, and impossible to quantify with all the interaction terms.

In our present extension of these earlier results, we initially confirmed that the effect of first breeding on future breeding was apparent in birds which differed in clutch size at first breeding. We then found that this effect did not differ among clutch-size groups. Taken at face value, our analysis suggests that laying a large number of eggs at first-breeding attempt is not more costly than laying fewer eggs, but that breeding per se is costly for young birds. In many other field studies of birds without manipulations, no evidence of trade-offs between clutch size and survival or capture rate was found (Nur, 1990). On the other hand, we could not demonstrate that this population is constituted of cohabiting 'good' and 'bad' birds, i.e. we did not find any positive correlation between clutch size and future breeding probability.

If either phenomenon exists in this population, we have probably failed to detect it because we worked with fewer data in each group than Viallefont et al. (1995). We also did not have enough data to work separately on each age class (birds starting to breed at age 2 or 3), even though we know that this is an important factor (Rockwell et al., 1993; Viallefont et al., 1995). This confounding probably reduced our power to detect an effect of clutch size. As shown in Section 6.1.4, 
the power of the test between models $\phi, p_{t+F}$ and $\phi, p_{t+F+F c l}$ was not sufficient to detect a small effect of clutch size on future reproduction. Thus, we think it likely that power for our actual test between models $\phi, p_{t+F+m}$ and $\phi, p_{t+F+m+F c l}$ was also low.

When possible, data requirements for such experiments, and a minimum level of detectable effect, should be evaluated before the start of the experiment, by power calculations. The capture rate should be high, or alternatively the number of individuals in the experiment should be great.

Of course, the use of appropriate survival estimates does not solve the problems of positive environmental or physiological correlations between reproduction and survival, and of the rapid energy turnover of 'income breeders'. On the contrary, these estimates can be used to detect positive correlation between reproduction and survival, or between subsequent reproductive events, as well as negative ones. As a result, both the hypotheses regarding costs of reproduction, or individual quality, can be tested, with properly settled experiments.

In this study, we were not able to use multi-state models, which would have enabled us to detect positive or negative correlations between clutch size across years. This is due to a lack of flexibility in the available computer programs which cannot handle the quite complex heterogeneity of capture in the data. We believe that most field studies present problems similar to those exemplified here, and that such costs of reproduction, if they exist, will be difficult to characterize due to a lack of data or a significant amount of heterogeneity in the data.

\section{Acknowledgements}

We thank Jean-Dominique Lebreton and the EURING94 meeting organisers for inviting us, and James Sedinger, James Nichols and an anonymous referee for very helpful comments on the manuscript. We also thank Jean-Dominique Lebreton for help with the power calculations.

Correspondence: A. Viallefont, CEFE, CNRS, BP 5051, F-34033 Montpellier Cedex 1, France.

\section{REFERENCES}

ARnAson, A. N. (1973) The estimation of population size, migration rates and survival in a stratified population, Researches on Population Ecology, 13, pp. 97-113.

Blondel, J., Pradel, R. \& Lebreton, J.-D. (1992) Low fecundity insular blue tits do not survive better than high fecundity mainland ones, fournal of Animal Ecology, 61, pp. 205-213.

Bradley, J. S., Wooller, R. D., Skira, I. J. \& Serventy, D. L. (1989) Age-dependent survival of breeding short-tailed shearwaters Puffinus tenuirostris, fournal of Animal Ecology, 58, pp. 175-188.

Burnham, K. P., Anderson, D. R., White, G. C., Brownie, C. \& Pollock, K. H. (1987) Design and Analysis Methods for Fish Survival Experiments Based on Release-Recapture (American Fisheries Society Monograph 5).

Cézilly, F., Viallefont, A., Boy, V. \& Johnson, A. R. (1996) Annual variation in survival and breeding probability in Greater Flamingos, Ecology (in press).

Clobert, J., Perrins, C. M., McCleery, R. H. \& Gosler, A. G. (1988) Survival rate in the Great Tit Parus major in relation to sex, age, and immigration status, fournal of Animal Ecology, 57, pp. 287-306.

Clobert, J., Lebreton, J.-D., Allaine, D. \& Gaillard, J. M. (1994) The estimation of age-specific breeding probabilities from recaptures or resightings in vertebrate populations: II. Longitudinal models, Biometrics, 50, pp. 375-387. 
DaAn, S., Dijkstra, C. \& Tinbergen, J. M. (1990) Family planning in the kestrel (Falco tinnunculus): The ultimate control of covariation of laying date and clutch size, Behaviour, 114, pp. 83-116.

Danchin, E. \& Monnat, J.-Y. (1992) Population dynamics modelling of two neighbouring Kittiwake, Rissa tridactyla colonies, Ardea, 80, pp. 171-180.

Findlay, C. S. \& CoOKe, F. (1987) Repeatability and heritability of clutch size in lesser snow geese, Evolution, 39, pp. 165-177.

Gadgil, M. \& Bossert, W. H. (1970) Life historical consequences of natural selection, American Naturalist, 106, pp. 258-261.

Gonzalez-Davilà, G. (1994) Démographie animale et Biologie Evolutive; modélisation et applications pur la gestion d'espèces sauvages, Thèse de doctorat ( $\mathrm{PhD}$ thesis), Université Montpellier II, Montpellier.

Gustaffson, L. \& Sutherland, W. J. (1988) The costs of reproduction in the collared flycatcher Ficedula albicollis, Nature, 335, pp. 813-815.

Hines, J. E. (1994) MSSURVIV, User's Manual (Laurel, MD, Patuxent Environmental Center).

Jönsson, K. I. \& Tuomi, J. (1994) Costs of reproduction in a historical perspective, Trends in Ecology and Evolution, 9, pp. 304-307.

Kendall, W. \& Nicholls, J. D. (1995) On the use of secondary capture-recapture samples to estimate temporary emmigration and breeding populations, fournal of Applied Statistics, 22, pp. $751-762$.

Lebreton, J.-D., Burnham, K. P., Clobert, J. \& Anderson, D. R. (1992) Modelling survival and testing biological hypotheses using marked animals: a unified approach with case studies, Ecological Monographs, 62, pp. 67-118.

Levins, R. (1968) Evolution in Changing Environments (Princeton, Princeton University Press).

Loison, A. (1995) Approaches intra- et inter-spécifiques de la dynamique des populations: l'example du chamois, PhD Thesis, Université Lyon.

Nichols, J. D., Hines, J. E., Pollock, K. H., Hinz, R. L. \& Link, W. A. (1994) A methodological framework for estimating breeding proportions and testing hypotheses about the cost of reproduction with capture-recapture data, Ecology, 75, pp. 2052-2065.

Newton, I. (1988) Age and reproduction in the Sparrowhawk. In: T. H. Clutton-Brock (Ed.), Reproductive Success, pp. 201-219 (Chicago, University of Chicago Press).

Nur, N. (1990) The cost of reproduction in birds: evaluating the evidence from manipulative and non-manipulative studies. In: J. Blondel, A. Gosler, J.-D. Lebreton \& R. McCleery (Eds), Population Biology of Passerine Birds: An Integrated Approach, pp. 281-296, NATO ASI Series, Series G: Ecological Sciences, Vol. 24 (Berlin, Springer Verlag).

Orell, M. \& Koivula, K. (1990) Effects of brood size manipulation of adult and juvenile survival and future fecundity in the Willow Tit Parus montanus. In: J. Blondel, A. Gosler, J.-D. Lebreton \& R. MCCleery (Eds), Population Biology of Passerine Birds: An Integrated Approach, pp. 297-306, NATO ASI Series, Series G: Ecological Sciences, Vol. 24 (Berlin, Springer Verlag).

Pollock, K. H. (1975) A K-sample tag-recapture model allowing for unequal survival and catchability, Biometrika, 62, pp. 577-583.

Pollock, K. H. (1981) Capture-recapture models allowing for age-dependent survival and capture rates, Biometrics, 37, pp. 521-529.

Pradel, R. (1993) Flexibility in survival analysis from recapture data: handling trap-dependence. In: J.-D. Lebreton \& P. M. North (Eds), Marked Individuals in the Study of Bird Populations, pp. 29-37 (Basel, Switzerland, Birkhaüser Verlag).

PRADEL, R. (1996) Utilisation of capture-mark-recapture for the study of recruitment and population growth rate, Biometrics (in press).

Pradel, R., Clobert, J. \& Lebreton, J.-D. (1990) Recent developments for the analysis of capture-recapture multiple data sets-an example concerning two Blue Tit populations, Ring, 13, pp. 193-204.

REZnick, D. (1985) Costs of reproduction: an evaluation of the empirical evidence, Oikos, 44, pp. 257-267.

Rockwell, R. F. (1989) Reproductive fitness in Snow Geese (Chen c. caerulescens): proximate and ultimate measures. In: Proceedings of XIXth International Ornithological Congress, pp. 2113-2120.

Rockwell, R. F., Cooch, E. G., Thompson, C. B. \& Cooke, F. (1993) Age and reproductive success in female lesser snow geese: experience, senescence and the cost of philopatry, fournal of Animal Ecology, 62, pp. 323-333.

Schwarz, C. J., Schweigert, J. F. \& Arnason, A. N. (1993) Estimating migration rates using tag-recovery data, Biometrics, 49, pp. 177-193. 
Sibly, R. M. \& Calow, P. (1986) Physiological Ecology of Animals (Oxford, Blackwell Scientific).

Skalski, J. R., Hoffman, A. \& Smith, S. G. (1993) Testing the significance of individual- and cohort-level covariates in animal survival studies. In: J.-D. Lebreton \& P. M. North (Eds), Marked Individuals in the Study of Bird Populations, pp. 9-28 (Basel, Switzerland, Birkhaüser Verlag).

Stearns, S. C. (1989) Trade-offs in life-history evolution, Functional Ecology, 3, pp. 259-268.

SteArns, S. C. (1992) The Evolution of Life Histories (Oxford, Oxford University Press).

Török, J. \& Tотн, L. (1990) Costs and benefits of reproduction in the Collared Flycatcher, Ficedula albicollis. In: J. Blondel, A. Gosler, J.-D. Lebreton \& R. MCCleery (Eds), Population Biology of Passerine Birds, pp. 307-319, NATO ASI Series, Series G: Ecological Sciences, Vol. 24 (Berlin, Springer Verlag).

Trivers, R. E. (1972) Parental investment and sexual selection. In: B. Campbell (Ed.), Sexual Selection and the Descent of Man, pp. 136-179 (Chicago, Aldine).

VAN NoordwiJk, A. J. \& DE Jong, G. (1986) Acquisition and allocation of resources: their influence on variation in life history tactics, American Naturalist, 128, pp. 137-142.

Viallefont, A. (1992) Robustesse et flexibilité des modélisations en capture-recapture; application aux oies des neiges (Anser caerulescens caerulescens), Mémoire de DEA, ENSA.M., Montpellier.

Viallefont, A., Cooke, F. \& Lebreton, J.-D. (1995) Age-specific costs of first-time breeding, $A u k$ (in press).

Weimerskirch, H. \& Jouventin, P. (1987) Population dynamics of the wandering albatross, Diomedea exulans, of the Crozet Island: causes and consequences of the population decline, Oikos, 49, pp. 315-322.

Williams, G. C. (1966) Natural selection, the cost of reproduction, and a refinement of Lack's principle, American Naturalist, 100, pp. 687-690. 
